\title{
(6) OPEN ACCESS \\ Social and economic conditions in childhood and the progression of functional health problems from midlife into old age
}

\author{
Neda Agahi, ${ }^{1}$ Benjamin A Shaw, ${ }^{2}$ Stefan Fors ${ }^{1}$
}

${ }^{1}$ Aging Research Center, Karolinska Institutet/Stockholm University, Stockholm, Sweden ${ }^{2}$ School of Public Health, University of Albany, State University of New York, New York, USA

\section{Correspondence to} Dr Neda Agahi,

Aging Research Center, Gävlegatan 16,

11330 Stockholm, Sweden; neda.agahi@ki.se

Received 29 November 2013 Revised 24 February 2014 Accepted 29 March 2014 Published Online First 23 April 2014

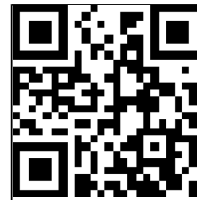

Open Access Scan to access more free content

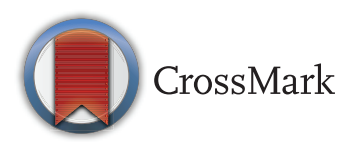

To cite: Agahi N, Shaw BA Fors S. J Epidemiol Community Health 2014;68:734-740.

\begin{abstract}
Background Childhood living conditions have been found to predict health and mortality in midlife and in old age. This study examines the associations between social and economic childhood conditions and the onset and progression of functional health problems from midlife into old age, and the extent to which potential associations are mediated by educational attainment and smoking.

Methods Data from the Level of Living Survey and the Swedish Panel Study of Living Conditions of the Oldest Old were merged to create a longitudinal data set with five repeated measures from 1968 to 2004 ( $n=1765$, aged 30-50 years and free from functional health problems at baseline). Multilevel regression models were used to analyse retrospective reports of social and economic conditions in childhood (eg, conflicts or economic problems in the family) in relation to the progression of functional health problems over the 36-year period.

Results Results showed that social and economic disadvantages in childhood were associated with an earlier onset and a faster progression of functional health problems from midlife into old age. Subsequent models showed that differences in educational attainment, but not smoking, explained much of the association between childhood disadvantages and trajectories of functional health problems.

Conclusions According to these results, adverse social and economic conditions in childhood affect the development of functional health problems from midlife into old age indirectly through less favourable life careers, including lower education. Creating equal opportunities for educational attainment may help reduce the long-term effects of disadvantaged childhood conditions and postpone functional health problems.
\end{abstract}

\section{INTRODUCTION}

Childhood living conditions have been repeatedly found to predict health throughout the life course. Numerous studies have reported associations between disadvantaged social and economic conditions during childhood and higher risks of physical and mental health problems, such as cardiovascular disease and depression, as well as mortality in adulthood and old age. ${ }^{1-8}$

Relatively few studies have investigated the association between childhood living conditions and later functional health problems, such as mobility impairment or disability. Preserving functional health is critical for the maintenance of independence and autonomy in midlife and old age, and for controlling the healthcare demands of aging populations. Results to date suggest that persons who have experienced social and economic disadvantages in childhood are at increased risk of developing mobility limitations and activity limitations in midlife, ${ }^{9-13}$ and spending a larger proportion of their lives with functional impairments. ${ }^{14}$ It is unclear whether these associations capture the effects of childhood disadvantages on later functional health, or rather the effects of long-standing health problems acquired during childhood on social, economic and functional disadvantages throughout the life course. ${ }^{15}$ In a recent study, childhood disadvantage and poor childhood health were associated with functional impairment and the estimated proportion of life spent with functional impairment. ${ }^{14}$ In the present study we use longitudinal data covering four decades to explore these associations further, by examining the onset and progression of functional health problems among persons with no functional impairments during midlife. By investigating the relationships between social and economic conditions in childhood and the onset and progression of new functional health problems from midlife into old age, the influence of prior health on later socioeconomic disadvantage is reduced, and we are better able to capture the effects of childhood conditions on the development of functional health problems later in life.

Social and economic hardships in childhood can have direct and indirect effects on later functional health. Exposures that affect development, and thereby have long-lasting health consequences, denote direct effects. For example, social disadvantages in childhood, such as conflicts in the family, are related to increased psychosocial stress which is hypothesised to affect cognitive development in childhood, ${ }^{16}{ }^{17}$ which in turn is related to functional ability. ${ }^{18}$ Economic disadvantages in childhood may have direct effects on functional health through the association between material deprivation and suboptimal nutrition and infectious diseases during gestation, infancy and childhood, which in turn affect general growth, including bone density and muscle mass, and thereby increase the risk of later functional health problems. ${ }^{19} 20$

Indirect effects imply that childhood conditions are part of chains of risk/advantage that set the individual on a certain pathway. As such, positive childhood conditions and experiences are associated with favorable events and behaviours later on in life, whereas disadvantaged conditions are associated with less favourable events and behaviours, that in turn lead to positive or negative 
health outcomes. ${ }^{21}$ Possible indirect mechanisms through which social and economic disadvantages in childhood affect later health and function may involve lower educational (or occupational) attainment and more unhealthy behaviours, such as smoking. ${ }^{1} 9102223$ These factors are associated with functional health problems, ${ }^{24} 25$ partly because of higher prevalence rates of lung problems, cardiovascular diseases and stroke among smokers and persons with lower education. ${ }^{26}{ }^{27}$ Further understanding of the association between childhood conditions and functional health problems, as well as potential mediators, can highlight mechanisms linking childhood conditions with midlife and late-life functional health, and lend insight into how chains of risk might be interrupted.

The aims of this study are twofold: first, to examine if social and economic conditions in childhood are related to the onset and long-term progression of functional health problems from midlife and into old age, and second, to investigate to what extent these potential associations can be attributed to differences in educational attainment and unhealthy behaviours (smoking) in midlife.

\section{MATERIAL AND METHODS Study sample}

Data came from the Swedish Level of Living Survey (LNU) and the Swedish Panel Study of Living Conditions of the Oldest Old (SWEOLD). LNU is a longitudinal, nationally representative, study based on a random sample of the Swedish adult population, ages 18-75 years. It was first carried out in 1968, and subsequently in 1974, 1981, 1991, 2000 and $2010 .^{28}$ Persons from the LNU sample who have passed the upper age limit of 75 years were included in SWEOLD. ${ }^{29}$ SWEOLD has been carried out in 1992, 2002, 2004 and 2011. In 2004, the lower age limit for inclusion in the study was 69 years. In both studies, professional interviewers conducted structured interviews with participants in their homes. In cases when the respondent could not participate due to health problems, proxy interviews were conducted instead. The interviews addressed questions about work life, family situation, childhood conditions, health behaviours, living conditions and health status. The Karolinska Institute Regional Research Ethics Committee reviewed and approved survey protocols.

Data from LNU 1968, 1981, 1991 and 2000 were merged with data from SWEOLD 2002 and 2004, which allowed for up to 36 years of follow-up. All individuals aged 30-50 years who were interviewed in 1968 were eligible for the study $(n=2051)$. Those reporting functional health problems at baseline or internal missing values $(n=286)$ were excluded from the analyses, resulting in 1765 individuals.

\section{Measurements}

Social and economic childhood living conditions (up to age 16 years) were measured retrospectively in 1968. Social aspects included conflicts in the childhood family and/or growing up without both biological parents. Respondents who were uncertain or answered 'Yes' to the question "Were there any serious conflicts in your family during your upbringing?" and/or answered 'No' to the question "Did you live with both your biological parents during your whole upbringing?" were categorised as having experienced social disadvantages during childhood. The economic aspect concerned financial hardships; respondents answering 'Yes' to the question "Did your family suffer from financial difficulties during your upbringing?" were categorised as having experienced economic disadvantages during childhood. These measures have been used previously in studies of the long-term health effects of childhood living conditions. $^{23}$

Smoking was measured in 1968 and 1981. In 1968, respondents were classified as current smokers or current non-smokers. In 1981, because some people in the sample had died or were non-responders, three categories were created: current smokers, current non-smokers and a third category for persons with missing information on smoking status. Non-responders and deceased persons in the additional smoking category did not differ with regard to childhood disadvantages, sex or education; but the deceased persons were older and more likely to be smokers in 1968. This made it possible to include all of the original respondents (from baseline, 1968) in the analyses.

Educational attainment was measured continuously as completed years of education.

Functional health was measured with indicators of mobility impairment collected in 1968, 1981, 1991, 2000/2002 and 2004. A summary index was constructed with responses to three items: the ability to walk 100 metres without difficulties, to run 100 metres without difficulties, and to go upstairs and downstairs without difficulties. Responses were 'yes' (0) and 'no' (1). The summed index ranged from no mobility problems (0) to problems in all three domains (3). This measure has been used previously to study the development of mobility problems from midlife to old age. ${ }^{24}$

Age was measured continuously in years (at baseline). Died during follow-up was a dichotomous variable indicating whether the respondent died during the 36-year follow-up period.

\section{Statistical analyses}

Individual-level changes in mobility impairment over time, measured as years since baseline, were modelled using multilevel models with occasions of measurement nested within individuals. $^{30}{ }^{31}$ Childhood conditions, years of education, smoking status as well as age, gender and death during follow-up were entered as time-fixed predictors of interindividual variation in the mobility impairment trajectories. Social and economic childhood disadvantages were analysed in separate sets of models when investigating the potential role of educational attainment and smoking in the association with the progression of functional health problems. Changes in functional health over time were estimated with linear multilevel regression including the quadratic function, which allowed us to estimate non-linear changes over time. Follow-up time, years since baseline, was centred; thus intercepts signify differences at the midpoint of the follow-up time rather than at baseline. Analyses were run using Hierarchical Linear Modelling software. ${ }^{32}$ Persons who died at some point during follow-up or were non-responders in any of the follow-up surveys were included in estimating the trajectories. In table 1 and figure 1 , comparisons are made with a 'No disadvantages' category that consists of persons who reported neither social nor economic disadvantages in childhood. In tables 2 and 3, the reference categories consist of persons who did not report social disadvantages (table 2) and those who did not report economic disadvantages (table 3), respectively.

\section{RESULTS}

Table 1 presents some of the characteristics of the sample as a whole, as well as divided according to childhood living conditions. A majority of the sample did not report any social or economic disadvantages in childhood (60.6\%). Economic disadvantages in childhood were reported by $28.4 \%$ and social disadvantages by $19.6 \% ; 8.7 \%$ reported social and economic 
Table 1 Descriptive characteristics of the study sample as a whole and divided by childhood disadvantage category ( $n=1765)$

\begin{tabular}{|c|c|c|c|c|}
\hline & No disadvantages $(n=1070)$ & Social disadvantages $(n=346)$ & Economic disadvantages $(n=502)$ & Total sample $(n=1765)$ \\
\hline \multicolumn{5}{|l|}{ Age } \\
\hline Mean age (SD) & $39.5(6.03)$ & $40.2(6.15)$ & $41.0(6.09)$ & $39.9(6.09)$ \\
\hline \multicolumn{5}{|l|}{ Sex } \\
\hline Women & 48.3 & 49.7 & 41.0 & 46.6 \\
\hline Men & 51.7 & 50.3 & 59.0 & 53.4 \\
\hline \multicolumn{5}{|l|}{ Years of education } \\
\hline Mean years of education (SD) & $8.86(3.25)$ & $8.40(3.02)$ & $7.75(2.49)$ & $8.54(3.09)$ \\
\hline \multicolumn{5}{|l|}{ Smoking } \\
\hline Non-smoker in 1968 & 55.5 & 52.0 & 52.2 & 54.0 \\
\hline Current smoker in 1968 & 44.5 & 48.0 & 47.8 & 46.0 \\
\hline Non-smoker in 1981 & 53.2 & 52.3 & 48.6 & 51.6 \\
\hline Current smoker in 1981 & 27.5 & 27.7 & 27.9 & 28.0 \\
\hline No smoking info in $1981 /$ dead & 19.3 & 19.9 & 23.5 & 20.5 \\
\hline Death during follow-up & 28.6 & 32.9 & 34.7 & 30.8 \\
\hline
\end{tabular}

disadvantages. Compared with those reporting no disadvantages, those reporting economic disadvantages in childhood were, on average, slightly older, had a higher proportion of men, fewer years of education and a higher proportion of respondents who died during the 36-year follow-up. Those reporting social disadvantages were generally somewhere in between.

Figure 1 illustrates the onset and progression of functional health problems from midlife and during the subsequent 36 years of follow-up leading up to old age, for the three childhood condition categories. The groups with social and/or economic disadvantages in childhood had an earlier onset and significantly more functional health problems during follow-up compared with those reporting no disadvantages, as also indicated by the coefficients for the intercepts in Model 1 in tables 2 and 3. As for the progression over time, there was an increase in mobility impairment during the follow-up period in all three groups, but persons with social and/or economic disadvantages had faster rates of increase in mobility problems from midlife into old age (see also the coefficients for the linear slopes in

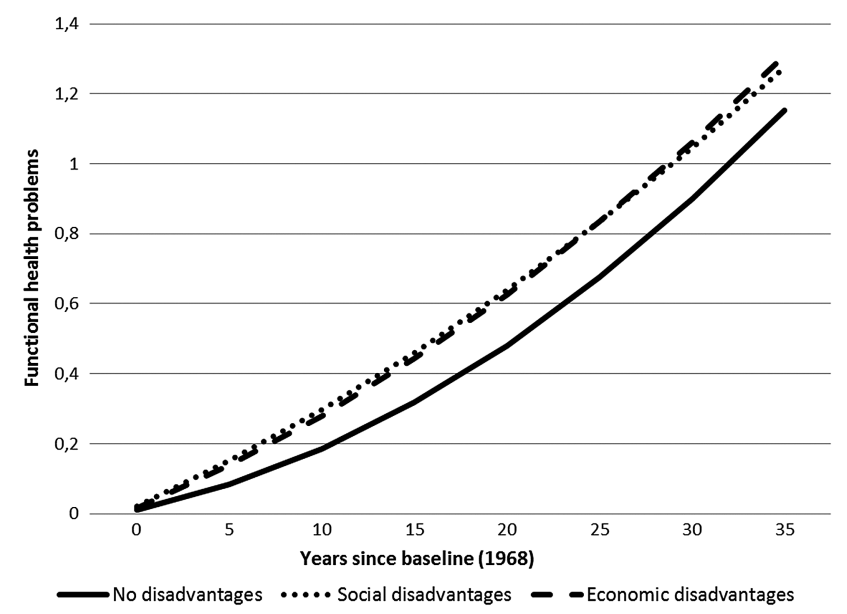

Figure 1 Association between social and economic conditions in childhood and the onset and progression of functional health problems from midlife into old age. Trajectories based on estimates from separate analyses and adjusted for age, sex and death during follow-up $(n=1765)$
Model 1, tables 2 and 3), compared with those reporting no such childhood disadvantages. For persons with social disadvantages in childhood, the onset and rate of progression of functional health problems exceeded that of persons with no such disadvantages during midlife, but the rate of acceleration in impairment during old age was slightly slower in this group (see coefficients for the quadratic slope in Model 1, table 2).

Models 2 to 4 in tables 2 and 3 investigated whether educational attainment and smoking behaviour mediated the associations of social and economic childhood disadvantages with trajectories of mobility impairment from midlife into old age. First, educational attainment was added to the model (Model 2). For social disadvantages (table 2) coefficients for the intercept did not change appreciably, and for economic disadvantages (table 3) estimates were lowered, but remained significant $(\mathrm{p} \leq 0.1)$. Differences in the progression of functional health problems (as indicated by the linear slopes), were no longer significant for the group reporting social disadvantages compared with those reporting no such disadvantages; although the lower rate of acceleration in the progression of problems during old age still remained. Estimates for economic disadvantages were substantially lowered, but remained significant at the $p \leq 0.1$ level.

Educational attainment in itself was significantly related to functional health problems; persons with higher education (more years of education) had lower levels as well as a slower progression of mobility impairment, although the quadratic slope indicated an accelerated progression of impairment in late life.

Model 3 investigated the effect of smoking behaviour on the association between social and economic disadvantages and trajectories of mobility impairment. Compared with Model 1, estimates for the level and progression of functional health problems for the social and the economic disadvantages groups remained unchanged when adding smoking behaviour to the model.

As for smoking behaviour, being a current smoker in 1968 (ages 30-50 years) was associated with more functional health problems as well as a faster progression from midlife to old age. Being a current smoker in 1981 (ages 43-63 years) was not associated with the level of impairment, but with a faster progression of problems as well as a late-life acceleration in the rate of progression, as indicated by the linear and quadratic slopes (Models 3 and 4, particularly in table 3).

When educational attainment and smoking behaviour were added to the regression model investigating the association 
Table 2 Associations between social disadvantages in childhood and functional health problems, and the role of educational attainment and smoking behaviour

\begin{tabular}{|c|c|c|c|c|c|c|c|c|c|c|}
\hline & \multicolumn{2}{|l|}{ Model 1} & \multicolumn{2}{|l|}{ Model 2} & \multicolumn{2}{|l|}{ Model 3} & \multicolumn{2}{|l|}{ Model 4} & \multicolumn{2}{|l|}{ Model 5‡ } \\
\hline & Coefficient & SE & Coefficient & SE & Coefficient & SE & Coefficient & SE & Coefficient & SE \\
\hline \multicolumn{11}{|l|}{ Fixed effects } \\
\hline Intercept & $0.337^{* * *}$ & 0.021 & $0.338^{* * *}$ & 0.021 & $0.272^{* * *}$ & 0.025 & $0.274 * * *$ & 0.025 & $0.258 * * *$ & 0.028 \\
\hline Social disadvantages & $0.122^{*}$ & 0.051 & $0.116^{*}$ & 0.050 & $0.118^{*}$ & 0.050 & $0.111^{*}$ & 0.050 & $0.098^{*}$ & 0.050 \\
\hline Educational attainment (years) & & & $-0.032^{* * *}$ & 0.005 & & & $-0.032^{* * *}$ & 0.005 & $-0.031 * * *$ & 0.005 \\
\hline \multicolumn{11}{|l|}{ Smoking } \\
\hline Current smoker in 1968 & & & & & $0.111^{*}$ & 0.053 & $0.116^{*}$ & 0.053 & $0.119 *$ & 0.053 \\
\hline Current smoker in 1981 & & & & & 0.025 & 0.058 & 0.017 & 0.057 & 0.015 & 0.058 \\
\hline Linear slope & $0.0308^{* * *}$ & 0.0014 & $0.0309 * * *$ & 0.0014 & $0.0255^{* * *}$ & 0.0016 & $0.0257 * * *$ & 0.0016 & $0.0250 * * *$ & 0.0016 \\
\hline Social disadvantages & $0.0033 t$ & 0.0021 & 0.0028 & 0.0020 & $0.0032 \dagger$ & 0.0020 & 0.0027 & 0.0020 & 0.0022 & 0.0020 \\
\hline Educational attainment (years) & & & $-0.0016 * * *$ & 0.0002 & & & $-0.0016 * * *$ & 0.0002 & $-0.0015^{* * *}$ & 0.0002 \\
\hline \multicolumn{11}{|l|}{ Smoking } \\
\hline Current smoker in 1968 & & & & & $0.0062 * *$ & 0.0022 & $0.0065 * *$ & 0.0022 & $0.0066^{* *}$ & 0.0022 \\
\hline Current smoker in 1981 & & & & & $0.0053^{*}$ & 0.0025 & $0.0048^{*}$ & 0.0025 & $0.0047 t$ & 0.0025 \\
\hline Quadratic slope & $0.00061 * * *$ & 0.00012 & $0.00061 * * *$ & 0.00012 & $0.00054^{* * *}$ & 0.00013 & $0.00054^{* * *}$ & 0.00013 & $0.00056^{* * *}$ & 0.00013 \\
\hline Social disadvantages & $-0.00028 t$ & 0.00017 & $-0.00028 t$ & 0.00017 & -0.00026 & 0.00017 & -0.00026 & 0.00017 & -0.00025 & 0.00017 \\
\hline Educational attainment (years) & & & $0.00004^{*}$ & 0.00002 & & & $0.00004^{*}$ & 0.00002 & $0.00004 t$ & 0.00002 \\
\hline \multicolumn{11}{|l|}{ Smoking } \\
\hline Current smoker in 1968 & & & & & -0.00007 & 0.00017 & -0.00008 & 0.00017 & -0.00008 & 0.00017 \\
\hline Current smoker in 1981 & & & & & 0.00029 & 0.00020 & 0.00030 & 0.00020 & 0.00030 & 0.00020 \\
\hline \multicolumn{11}{|l|}{ Random effects } \\
\hline \multicolumn{11}{|l|}{ Variances } \\
\hline Intercept & $0.172^{* * *}$ & & $0.166^{* * *}$ & & $0.168^{* * *}$ & & $0.161 * * *$ & & $0.161 * * *$ & \\
\hline Linear slope & $0.0007^{* * *}$ & & $0.0007^{* * *}$ & & $0.0007^{* * *}$ & & $0.0006^{* * *}$ & & $0.0006^{* * *}$ & \\
\hline
\end{tabular}

Coefficients from multilevel regressions. All models are adjusted for age, sex and death during follow-up ( $n=1765)$.

$t p \leq 0.1,{ }^{*} p \leq 0.05,{ }^{* *} p \leq 0.01,{ }^{* * *} p \leq 0.001$.

¥Additionally adjusts for economic childhood disadvantages, coefficients for economic disadvantages can be found in table 3 , Model 5 .

between social disadvantages and functional health problems (Model 4, table 2), only the association with level of functional health problems remained significant. For economic disadvantages (Model 4, table 3), the level and the progression of problems were significant $(p \leq 0.1)$. Coefficients for educational attainment and smoking behaviour remained more or less unchanged compared with previous models.

Finally, estimates were lowered for social and economic childhood disadvantages in Model 5 (in tables 2 and 3) when these two variables were entered into the same model. While the level of functional health problems remained significantly higher for the social disadvantages group, neither the level nor the progression of problems was significantly higher in the group reporting economic disadvantages.

\section{DISCUSSION}

These results add to previous studies by showing an association between adverse childhood living conditions and the onset and progression of functional health problems from midlife into old age. Persons who reported social and economic disadvantages in childhood had a higher level of mobility impairment during the follow-up period, and also a faster progression of problems compared with those reporting no childhood disadvantages. While previous studies have shown that adults who experienced adverse childhood conditions tend to have relatively poor health and higher rates of mortality in midlife as well as in old age, the current study indicates that these individuals actually experience an earlier onset and faster progression of functional health problems from midlife into old age.
Previous research has suggested that selection processes may explain part of the association between childhood conditions and later health. ${ }^{15} \mathrm{~A}$ strength of our study, however, was its ability to capture the onset and progression of 'new' functional health problems-rather than capturing the effects of longstanding health problems on social, financial and functional disadvantages throughout the life course-by excluding persons with functional health problems at baseline (in midlife, ages 30-50 years).

Further analyses investigating the mediating role of educational attainment and smoking suggest that education, but not smoking, accounts for most of the association between childhood conditions and the development of functional health problems. Among those reporting economic disadvantages, the higher level and the faster progression of functional health problems seem to be largely explained by differences in educational attainment, suggesting indirect effects. Among those reporting social disadvantages, associations were less affected after accounting for educational attainment and smoking, but the progression rate was no longer statistically different from those reporting no childhood disadvantages. The persisting higher level of functional health problems among persons with social disadvantages suggests that other factors than educational attainment and smoking are involved in the pathway between adverse childhood conditions and later functional health problems.

In this study, social and economic disadvantages in childhood were primarily analysed separately, but their effects are hard to differentiate as they are often interrelated; financial hardships increase parental strain and the risk of conflicts in the family and can thereby influence the psychosocial environment in the 
Table 3 Associations between economic disadvantages in childhood and functional health problems, and the role of educational attainment and smoking behaviour

\begin{tabular}{|c|c|c|c|c|c|c|c|c|c|c|}
\hline & \multicolumn{2}{|l|}{ Model 1} & \multicolumn{2}{|l|}{ Model 2} & \multicolumn{2}{|l|}{ Model 3} & \multicolumn{2}{|l|}{ Model 4} & \multicolumn{2}{|l|}{ Model $5 \ddagger$} \\
\hline & Coefficient & SE & Coefficient & SE & Coefficient & SE & Coefficient & SE & Coefficient & SE \\
\hline \multicolumn{11}{|l|}{ Fixed effects } \\
\hline Intercept & $0.330 * * *$ & 0.022 & $0.338 * * *$ & 0.022 & $0.263 * * *$ & 0.027 & $0.272 * * *$ & 0.027 & $0.258 * * *$ & 0.028 \\
\hline Economic disadvantages & $0.112 * *$ & 0.044 & $0.082 t$ & 0.044 & $0.111^{*}$ & 0.044 & $0.080 t$ & 0.044 & 0.064 & 0.044 \\
\hline Educational attainment (years) & & & $-0.031 * * *$ & 0.005 & & & $-0.031 * * *$ & 0.005 & $-0.031 * * *$ & 0.005 \\
\hline \multicolumn{11}{|l|}{ Smoking } \\
\hline Current smoker in 1968 & & & & & $0.121^{*}$ & 0.053 & $0.125^{*}$ & 0.053 & $0.119 *$ & 0.053 \\
\hline Current smoker in 1981 & & & & & 0.054 & 0.058 & 0.009 & 0.058 & 0.015 & 0.058 \\
\hline Linear slope & $0.0301 * * *$ & 0.0015 & $0.0306 * * *$ & 0.0014 & $0.0248 * * *$ & 0.0016 & $0.0253 * * *$ & 0.0016 & $0.0250 * * *$ & 0.0016 \\
\hline Economic disadvantages & $0.0047 * *$ & 0.0018 & $0.0031 t$ & 0.0018 & $0.0046^{*}$ & 0.0018 & $0.0031 t$ & 0.0018 & 0.0027 & 0.0018 \\
\hline Educational attainment (years) & & & $-0.0015^{* * *}$ & 0.0002 & & & $-0.0015^{* * *}$ & 0.0002 & $-0.0015^{* * *}$ & 0.0002 \\
\hline \multicolumn{11}{|l|}{ Smoking } \\
\hline Current smoker in 1968 & & & & & $0.0065 * *$ & 0.0022 & $0.0067 * *$ & 0.0022 & $0.0066^{* *}$ & 0.0022 \\
\hline Current smoker in 1981 & & & & & $0.0049 *$ & 0.0025 & $0.0046 t$ & 0.0025 & $0.0047 t$ & 0.0025 \\
\hline Quadratic slope & $0.00060^{* * *}$ & 0.00012 & $0.00059 * * *$ & 0.00012 & $0.00053^{* * *}$ & 0.00013 & $0.00052^{* * *}$ & 0.00013 & $0.00056^{* * *}$ & 0.00013 \\
\hline Economic disadvantages & -0.00017 & 0.00015 & -0.00014 & 0.00015 & -0.00017 & 0.00015 & -0.00013 & & -0.00009 & 0.00015 \\
\hline Educational attainment (years) & & & $0.00004 \dagger$ & 0.00002 & & & $0.00004 \dagger$ & 0.00002 & $0.00004 t$ & 0.00002 \\
\hline \multicolumn{11}{|l|}{ Smoking } \\
\hline Current smoker in 1968 & & & & & -0.00009 & 0.00018 & -0.00010 & 0.00018 & -0.00008 & 0.00017 \\
\hline Current smoker in 1981 & & & & & $0.00031 t$ & 0.00020 & $0.00031 t$ & 0.00020 & 0.00030 & 0.00020 \\
\hline \multicolumn{11}{|l|}{ Random effects } \\
\hline \multicolumn{11}{|l|}{ Variances } \\
\hline Intercept & $0.172^{* * *}$ & & $0.166^{* * *}$ & & $0.167^{* * *}$ & & $0.161 * * *$ & & $0.161 * * *$ & \\
\hline Linear slope & $0.0007^{* * *}$ & & $0.0007^{* * *}$ & & $0.0007^{* * *}$ & & $0.0006^{* * *}$ & & $0.0006^{* * *}$ & \\
\hline
\end{tabular}

Coefficients from multilevel regressions. All models are adjusted for age, sex and death during follow-up ( $n=1765)$.

$t p \leq 0.1,{ }^{*} p \leq 0.05,{ }^{* *} p \leq 0.01,{ }^{* * *} p \leq 0.001$

¥Additionally adjusts for social childhood disadvantages, coefficients for social disadvantages can be found in table 2, Model 5 .

home negatively. ${ }^{16}$ Nine per cent of the sample reported social and economic disadvantages. The final model in the analyses included mutual adjustment for social and economic disadvantages, and revealed that social disadvantages-but not economic disadvantages-were still significantly related to the level of functional health problems from midlife to old age. This may imply that the association between childhood economic disadvantages and the progression of functional health problems is to some extent due to concurrent social problems. This is in line with studies suggesting that it is the accumulation of adverse socioeconomic exposures in childhood, rather than the specific exposures per se, that make childhood conditions a strong predictor of later health. ${ }^{14}$

Regardless of whether adverse childhood conditions set the individual on an unfavourable life path or whether they initiate irreversible physiological processes that will become manifest years later, the present results suggest that material deprivation as well as the psychosocial environment in childhood have longlasting effects on aging and functional health decades later. Similar to the results of this study, Tubeuf and colleagues ${ }^{1}$ found that financial hardships and the absence of a father during childhood were associated with impaired midlife health, but that most of the association between childhood conditions and midlife health was mediated by educational attainment and lifestyle factors such as smoking, obesity and alcohol habits. Health behaviours other than smoking were not included in the present study as they were very crudely measured (or not measured at all) at baseline in 1968 .

The negligible effect of smoking on the association between childhood conditions and functional health problems in the present study was unexpected. A possible explanation is that smoking was not particularly stratified in these cohorts, as presented in table 1 and also shown in another study of the same birth cohorts. ${ }^{33}$ In later cohorts smoking seems to be more socially stratified. ${ }^{34}$ Thus, smoking may explain more of the association between childhood conditions and later health and function in later cohorts, in line with other studies showing stronger associations between childhood conditions and later health behaviours. ${ }^{22} 23$

\section{Strengths and limitations}

The strengths of this study are the nationally representative sample, the high response rates and the repeated measures over a period of 36 years allowing for the estimation of long-term trajectories of mobility impairment; something that has not been done previously. Results can be generalised due to the randomly selected national sample and the high response rates.

As for limitations, this study, as well as many other studies about childhood conditions, relies on retrospective data on childhood conditions, with the problems of recall error and recall bias that may come with it. According to one study, the use of retrospective reports may underestimate results as compared with historical information from school/nurse records. ${ }^{35}$ In addition, the measurement of childhood conditions is subjective and rather unspecific; for example, what is considered a 'serious conflict' is up to the respondent. Although this makes the question rather vague, it also makes it more inclusive as no types of conflicts are excluded. Still, this study as well as earlier studies using these measures, have found associations with various indicators of later morbidity. ${ }^{2}{ }^{3}$ Selective survival is 
another factor to consider when studying associations between adverse conditions early in life and later life health outcomes, since mortality is higher in disadvantaged groups. Persons who survive into old age are therefore more advantaged, healthier and more robust to the consequences of aging compared with those that die, which may bias the results. ${ }^{36}$ In the present study, the whole baseline sample of 30-50-year-olds was included in the analyses and contributed to the estimation of trajectories of mobility impairment. Death at any point during the follow-up period was included as a control variable.

In conclusion, this study confirms and expands previous study results by showing that social and economic disadvantages in childhood are related to an earlier onset and faster progression of functional health problems from midlife to old age. Associations between childhood disadvantages and later functional health problems are partly explained by differences in educational attainment, suggesting indirect effects working through less favourable life careers, including lower education, among persons who experienced adverse social and economic conditions in childhood. ${ }^{21}{ }^{36}$ Creating equal opportunities for educational attainment may help reduce the long-term effects of disadvantaged childhood conditions by postponing functional health problems, and ultimately helping to control the healthcare demands of aging populations.

\section{What is already known on this subject?}

- Childhood living conditions have been repeatedly found to predict health throughout the life course, but few studies have investigated the association with later functional health problems.

- Preserving functional health is critical for the maintenance of independence and autonomy in midlife and old age.

- Further understanding of the association can highlight mechanisms linking childhood conditions with later functional health, and lend insight into how chains of risk might be interrupted.

\section{What this study adds?}

- Social and economic disadvantages in childhood are related to an earlier onset and faster progression of functional health problems from midlife to old age.

- Associations between childhood disadvantages and later functional health problems are partly explained by differences in educational attainment.

- Creating equal opportunities for educational attainment may help reduce the long-term effects of disadvantaged childhood conditions by postponing functional health problems, and ultimately helping to control the healthcare demands of aging populations.

Contributors NA and SF designed the study. NA and BAS conducted the statistical analyses. All contributed to the interpretation of results. NA wrote the manuscript. SF and BAS assisted in drafting the manuscript and revising it critically for intellectual content. All approved the final version of the manuscript to be published.

Funding This work was supported by Forte, the Swedish Research Council for Health, Working Life and Welfare (2010-0954 to NA and 2012-0761 to SF).

Competing interests None.
Ethics approval The Karolinska Institute Regional Research Ethics Committee. Provenance and peer review Not commissioned; externally peer reviewed.

Open Access This is an Open Access article distributed in accordance with the Creative Commons Attribution Non Commercial (CC BY-NC 3.0) license, which permits others to distribute, remix, adapt, build upon this work non-commercially, and license their derivative works on different terms, provided the original work is properly cited and the use is non-commercial. See: http://creativecommons.org/ licenses/by-nc/3.0/

\section{REFERENCES}

1 Tubeuf $S$, Jusot F, Bricard D. Mediating role of education and lifestyles in the relationship between early-life conditions and health: evidence from the 1958 British cohort. Health Econ 2012;21(Suppl 1):129-50.

2 Lundberg 0 . The impact of childhood living conditions on illness and mortality in adulthood. Soc Sci Med 1993;36:1047-52.

3 Fors $\mathrm{S}$, Lennartsson $\mathrm{C}$, Lundberg $\mathrm{O}$. Childhood living conditions, socioeconomic position in adulthood, and cognition in later life: exploring the associations. J Gerontol B Psychol Sci Soc Sci 2009;64:750-7.

4 Shaw BA, Krause N, Chatters LM, et al. Emotional support from parents early in life, aging, and health. Psychol Aging 2004;19:4-12.

5 Galobardes B, Lynch JW, Davey Smith G. Childhood socioeconomic circumstances and cause-specific mortality in adulthood: systematic review and interpretation. Epidemiol Rev 2004;26:7-21.

6 Schafer MH, Ferraro KF. Childhood misfortune and adult health: enduring and cascadic effects on somatic and psychological symptoms? J Aging Health 2013;25:3-28.

7 Kelly-Irving M, Lepage B, Dedieu D, et al. Adverse childhood experiences and premature all-cause mortality. Eur J Epidemiol 2013;28:721-34.

8 Galobardes B, Smith GD, Lynch JW. Systematic review of the influence of childhood socioeconomic circumstances on risk for cardiovascular disease in adulthood. Ann Epidemiol 2006;16:91-104.

9 Osler M, Madsen M, Nybo Andersen AM, et al. Do childhood and adult socioeconomic circumstances influence health and physical function in middle-age? Soc Sci Med 2009;68:1425-31.

10 Elstad J. Childhood adversities and health variations among middle-aged men: a retrospective lifecourse study. Eur J Public Health 2005;15:51-8.

11 Strand BH, Cooper R, Hardy R, et al. Lifelong socioeconomic position and physical performance in midlife: results from the British 1946 birth cohort. Eur J Epidemiol 2011;26:475-83

12 Birnie K, Cooper R, Martin RM, et al. Childhood socioeconomic position and objectively measured physical capability levels in adulthood: a systematic review and meta-analysis. PLoS One 2011;6:e15564

13 Birnie K, Martin RM, Gallacher J, et al. Socio-economic disadvantage from childhood to adulthood and locomotor function in old age: a lifecourse analysis of the Boyd Orr and Caerphilly prospective studies. J Epidemiol Community Health 2011;65:1014-23.

14 Montez JK, Hayward MD. Cumulative Childhood Adversity, Educational Attainment and Active Life Expectancy Among U.S. Adults. Demography 2013;51:413-35.

15 Deaton $A$. What does the empirical evidence tell us about the injustice of health inequalities? In: Eyal N, Hurst SA, Norheim OF, Wikler D, eds. Inequalities in Health: Concepts, Measures, and Ethics. New York: Oxford University Press, 2013:263-81.

16 Blair C, Raver CC. Child development in the context of adversity: experiential canalization of brain and behavior. Am Psychol 2012;67:309-18.

17 Danese A, McEwen BS. Adverse childhood experiences, allostasis, allostatic load, and age-related disease. Physiol Behav 2012;106:29-39.

18 Wahl HW, Schmitt M, Danner D, et al. Is the emergence of functional ability decline in early old age related to change in speed of cognitive processing and also to change in personality? J Aging Health 2010;22:691-712.

19 Rizzoli R, Bianchi ML, Garabedian M, et al. Maximizing bone mineral mass gain during growth for the prevention of fractures in the adolescents and the elderly. Bone 2010;46:294-305.

20 Patel HP, Jameson KA, Syddall HE, et al. Developmental influences, muscle morphology, and sarcopenia in community-dwelling older men. J Gerontol A Biol Sci Med Sci 2012;67:82-7.

21 Kuh D, Power C, Blane D, et al. Socioeconomic pathways between childhood and adult health. In: Kuh D, Ben-Shlomo Y, eds. A life course approach to chronic disease epidemiology. 2nd ed. Oxford: Oxford university press, 2004:371-95.

22 Osler M, Godtfredsen NS, Prescott E. Childhood social circumstances and health behaviour in midlife: the Metropolit 1953 Danish male birth cohort. Int J Epidemiol 2008;37:1367-74.

23 Jefferis BJ, Power C, Graham $\mathrm{H}$, et al. Effects of childhood socioeconomic circumstances on persistent smoking. Am J Public Health 2004;94:279-85.

24 Agahi N, Shaw BA. Smoking trajectories from midlife to old age and the development of non-life-threatening health problems: a 34-year prospective cohort study. Prev Med 2013;57:107-12. 
25 Coppin AK, Ferrucci L, Lauretani F, et al. Low socioeconomic status and disability in old age: evidence from the InChianti study for the mediating role of physiological impairments. J Gerontol A Biol Sci Med Sci 2006;61:86-91.

26 U.S. Department of Health and Human Services. The Health Consequences of Smoking: A Report of the Surgeon General. Atlanta, GA: U.S. Department of Health and Human Services, Centers for Disease Control and Prevention, National Center for Chronic Disease Prevention and Health Promotion, Office on Smoking and Health, 2004.

27 Dalstra JA, Kunst AE, Borrell $C$, et al. Socioeconomic differences in the prevalence of common chronic diseases: an overview of eight European countries. Int J Epidemiol 2005;34:316-26.

28 Fritzell J, Lundberg 0. Health Inequalities and Welfare Resources. Bristol: Policy Press, 2007.

29 Lundberg 0 , Thorslund M. Fieldwork and measurement considerations in surveys of the oldest old. Experiences from the Swedish level of living surveys. Social Indicators Research 1996:37:165-89.
30 Hox J. Multilevel Analysis: Techniques and Applications. Mahwah, NJ: Lawrence Erlbaum Associates, 2002.

31 Shaw BA, Liang J. Growth Models with Multilevel Regression. In: Newsom JT, Jones RN, Hofer SM, eds. Longitudinal Data Analysis A Practical Guide for Researchers in Aging, Health, and Social Sciences. London: Routledge Academic, 2011:217-42.

32 Raudenbush SW, Bryk AS. Hierachical linear models. Thousand Oaks, CA: Sage, 2002

33 Fors S, Agahi N, Shaw BA. Paying the price? The impact of smoking and obesity on health inequalities in later life. Scand J Public Health 2013:41:134-41.

34 Norberg M, Lundqvist G, Nilsson $M$, et al. Changing patterns of tobacco use in a middle-aged population: the role of snus, gender, age, and education. Glob Health Action Published Online First: 3 Jun 2011 doi:10.3402/gha.v4i0.5613.

35 Kauhanen L, Lakka HM, Lynch JW, et al. Social disadvantages in childhood and risk of all-cause death and cardiovascular disease in later life: a comparison of historical and retrospective childhood information. Int J Epidemiol 2006:35:962-8.

36 Ferraro KF, Shippee TP. Aging and cumulative inequality: how does inequality get under the skin? Gerontologist 2009;49:333-43. 